Zbl Arbeitsmed 2017 $\cdot 67: 151-154$ DOI 10.1007/s40664-017-0182-4 Online publiziert: 18. April 2017 (c) Der/die Autor(en) 2017. Dieser Artikel ist eine Open-Access-Publikation. CrossMark

M. Nübling $\cdot$ M. Vomstein $\cdot$ A. Haug $\cdot$ H.-J. Lincke

Freiburger Forschungsstelle für Arbeitswissenschaften GmbH (FFAW), Freiburg, Deutschland

\title{
Sind Referenzdaten der COPSOQ- Datenbank für eine JEM zu psychosozialen Arbeitsfaktoren geeignet?
}

\section{Hintergrund und Fragestellung}

Eine Job-Expositions-Matrix (JEM) versucht, eine externe Beurteilung von Belastungen („exposures“) anhand von „jobs“ (Berufen, Tätigkeiten) vorzunehmen.

Ziel ist es, Belastungen möglichst genau abzuschätzen, ohne eine direkte Inaugenscheinnahme oder Analyse des konkreten Arbeitsplatzes vornehmen zu müssen, d.h. die wahrscheinliche Belastung aus der Tätigkeit zu prognostizieren.

Die Güte einer JEM steht und fällt mit der Treffsicherheit dieser Vorhersage. Methodisch gesehen ist eine JEM umso besser, je mehr Varianz der Exposition durch die Tätigkeit erklärt werden kann, d. h. der Tätigkeit zugeordnet werden kann. Mitglieder einer Tätigkeitsgruppe sollten dann bezüglich der Exposition möglichst homogen sein („variation within group“). Die Verschiedenheit hinsichtlich der Exposition oder Belastung sollte v. a. zwischen den Berufsgruppen liegen („variation between groups“).

Im Themenfeld physische Faktoren und Berufe haben JEM eine lange und erfolgreiche Tradition, z. B. kommen Expositionen wie Heben und Tragen, Mehlstaub, biologische Arbeitsstoffe etc. in manchen Berufsgruppen deutlich häufiger vor als in anderen. In der Regel sind diese JEM umso besser, je genauer sich die Exposition charakterisieren lässt (z. B. Mehlstaub statt Staub) und je detaillierter sich die Tätigkeit beschreiben lässt (z. B. Bäcker statt Lebensmittelberufe), weil dann sowohl „exposure“ als auch „job“ innerhalb dieser Definitionsgrenzen homogener werden (s. dazu weitere Artikel zum Thema JEM in diesem Heft).

Die Fragestellung der vorliegenden Untersuchung ist, ob und inwieweit eine solche Zuordnung auch bei psychischen Belastungen und Gefährdungen gelingen kann.

\section{Studiendesign und Unter- suchungsmethoden}

Der Fragebogen COPSOQ (Copenhagen Psychosocial Questionnaire; [3]) ist ein umfassender Fragebogen zur Erhebung eines differenzierten Bildes der psychosozialen Faktoren bei der Arbeit. Die deutsche Standardversion [5] beinhaltet
19 „Belastungen“ (psychosoziale Situation am Arbeitsplatz, positiv wie negativ, „Ursachen“) und 6 „Beanspruchungen“ (Reaktion der Person, positiv wie negativ, „Folgen“).

- Abb. 1 zeigt die 25 Konstrukte, gegliedert nach Anforderungen, Einfluss und Entwicklungsmöglichkeiten, soziale Beziehungen und Führung, Unsicherheit des Arbeitsplatzes und den Belastungsfolgen im Bereich Zufriedenheit und Gesundheit.

Aus der COPSOQ-Datenbank der FFAW wurden 10.000 berufsrepräsentative Fälle ausgewählt. In Varianzanalysen wurde geprüft, inwieweit jeder der 25 COPSOQ-Aspekte mit der Berufsgruppe (Klassifikation der Berufe nach KldB92, nur Gruppen mit mindes-

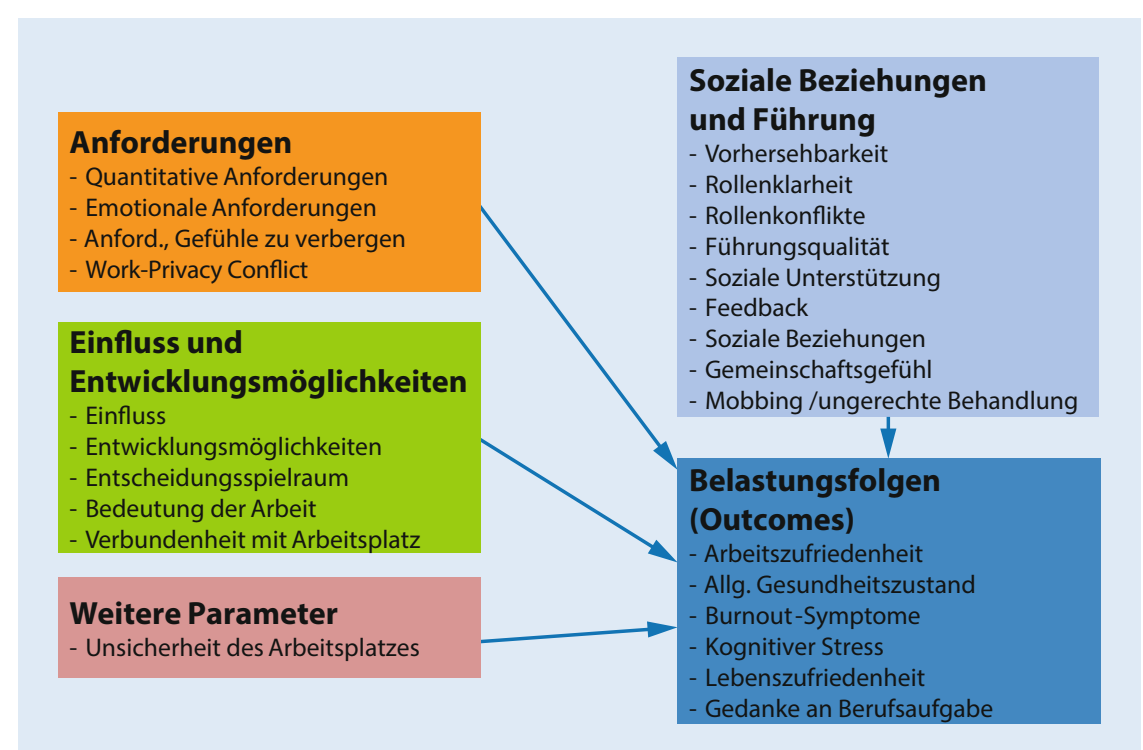

Abb. 1 ^ COPSOQ (deutsche Standardversion) 


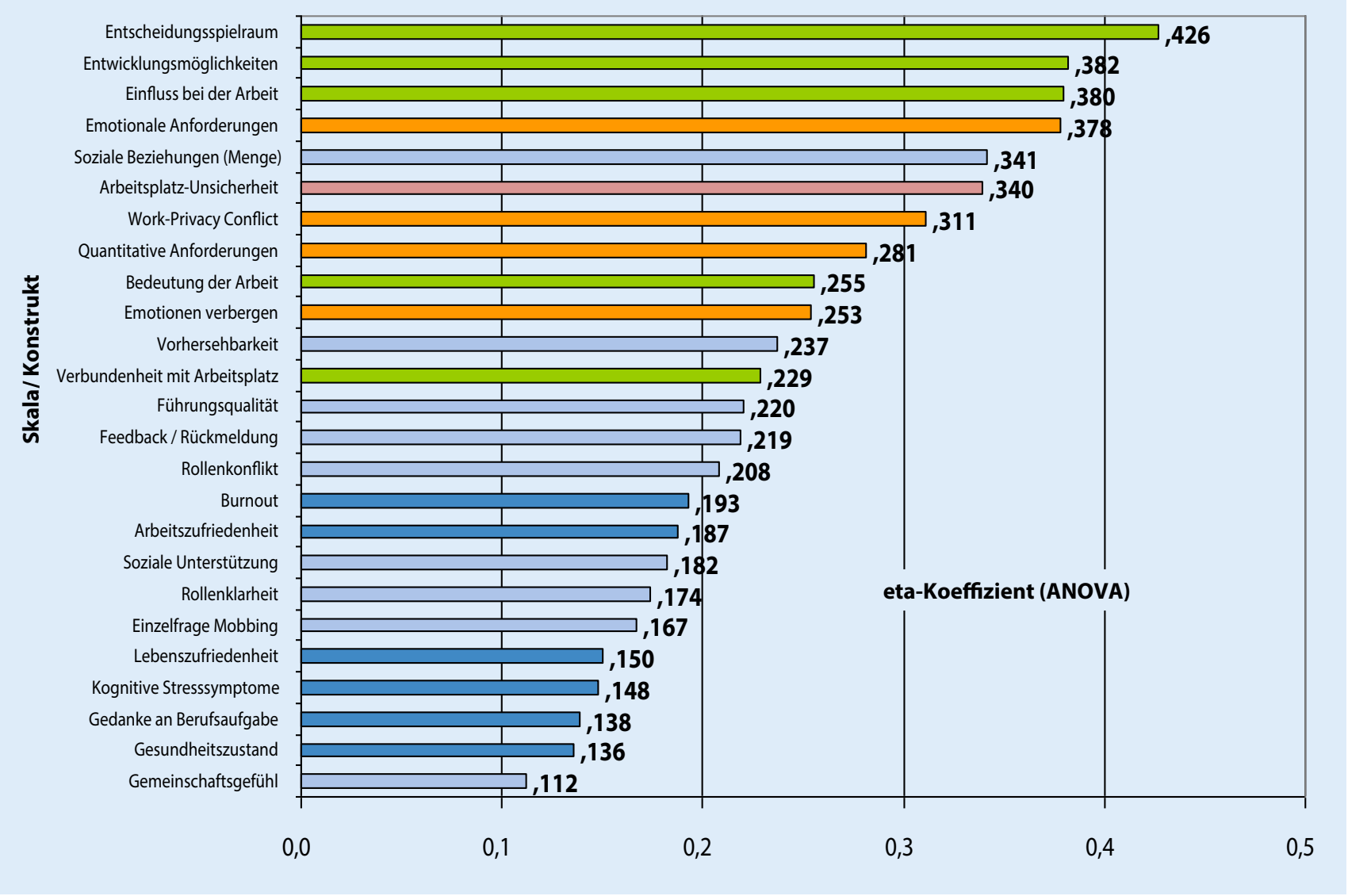

Abb. 2 ^ Zusammenhänge COPSOQ-Skalen mit Berufsgruppen (KldB92)

tens 30 Personen) zusammenhängt. Der eta-Koeffizient (Wertebereich $0-1$, eta ${ }^{2}$ wäre der prozentuale Anteil der durch die Berufsgruppe erklärten Varianz) zeigt dabei das Ausmaß des Zusammenhangs von Berufsgruppe (,job“) und Arbeitsplatzfaktor („exposure“). Je höher eta $\left(\right.$ oder eta $\left.{ }^{2}\right)$ ist, desto stärker wird die Exposition durch die Berufsgruppe beeinflusst und desto treffsicherer wäre eine Prädiktion in einer JEM.

\section{Ergebnisse}

In $\bullet$ Abb. 2 sind die eta-Koeffizienten der Varianzanalysen der Berufsgruppe mit jedem der 25 COPSOQ-Aspekte angegeben. Zur besseren Orientierung wiederholt die Farbgebung die Eingruppierung aus $\bullet$ Abb. 1.

Es wird deutlich, dass die eta-Koeffizienten je nach Thema relativ stark variieren; sie liegen zwischen moderaten Werten um die 0,4 bis zu schwachen Zusammenhangswerten von 0,1 . Die stärksten
Zusammenhänge mit der Tätigkeit zeigen Aspekte aus dem Feld Einfluss und Entwicklungsmöglichkeiten: 0,42 für Entscheidungsspielraum, 0,38 für Entwicklungsmöglichkeiten und 0,38 für Einfluss bei der Arbeit. Die Aspekte zu Anforderungen und die Unsicherheit des Arbeitsplatzes finden sich im Mittelfeld mit Zusammenhangsziffern zwischen 0,37 und 0,25. Am schwächsten hängen die Aspekte aus dem Bereich soziale Beziehungen und Führung (eta in der Regel etwa 0,2) und die Belastungsfolgen (eta-Koeffizienten zwischen 0,14 und 0,19) mit der Berufsgruppe zusammen.

Das heißt, psychosoziale Arbeitsplatzfaktoren sind je nach Thema unterschiedlich stark berufsassoziiert, die Zusammenhänge liegen im moderaten bis schwachen Bereich.

\section{Diskussion}

Die Ausprägung einiger psychosozialer Faktoren, wie z. B. Einfluss oder Entschei- dungsspielraum, weist noch einen relativ deutlichen Zusammenhang zur Berufsgruppe auf. Bei einer differenzierten Erhebung der psychosozialen Faktoren können also einige durchaus in Form einer JEM abgebildet werden.

Je gröber das Konstrukt „psychosoziale Belastungen“ operationalisiert wird, je stärker also unterschiedliche Belastungsfaktoren wie z. B. Einfluss, emotionale Anforderungen und Führungsqualität zusammengefasst werden, desto geringer wird die Erklärkraft durch den Beruf oder die Tätigkeit. Kroll [4] konstatiert z. B. anhand eines summarischen Index für psychische Belastungen, dass sich diese - im klaren Gegensatz zu den physischen Belastungen - wenig für das Verfahren der JEM eignen.

Bei vielen psychosozialen Faktoren ist dagegen kaum ein Zusammenhang zur Berufsgruppe gegeben, insbesondere für die Aspekte im Feld: soziale Beziehungen und Führung (• Abb. 2). 
Zbl Arbeitsmed 2017·67:151-154 DOI 10.1007/s40664-017-0182-4

(c) Der/die Autor(en) 2017. Dieser Artikel ist eine Open-Access-Publikation.

M. Nübling · M. Vomstein · A. Haug · H.-J. Lincke

\section{Sind Referenzdaten der COPSOQ-Datenbank für eine JEM zu psychosozialen Arbeitsfaktoren geeignet?}

\section{Zusammenfassung}

Hintergrund. Die Beurteilung von Belastungen („exposures") anhand von Berufen oder Tätigkeiten in einer Job-ExpositionsMatrix (JEM) ist in vielen Bereichen der Arbeitsepidemiologie ein bewährtes Verfahren.

Ziel der Arbeit. Die vorliegende Untersuchung prüft, ob und inwieweit eine solche Zuordnung auch bei psychosozialen Belastungen und Gefährdungen gelingen kann.

Material und Methoden. Mit 10.000

berufsrepräsentativen Fällen aus der COPSOQDatenbank wurde geprüft, inwieweit die Varianz in den 25 Konstrukten zu den Themen Anforderungen, Einfluss und Entwicklungsmöglichkeiten, soziale Beziehungen und Führung, Unsicherheit des Arbeitsplatzes und
Belastungsfolgen durch die Berufsgruppe (KldB92) erklärt werden kann.

Ergebnisse. Psychosoziale Arbeitsplatzfaktoren sind je nach Themenfeld unterschiedlich stark berufsassoziiert, die Zusammenhänge liegen im moderaten bis schwachen Bereich (eta $=0,11-0,43$ ).

Im Bereich Einfluss und Entwicklungsmöglichkeiten werden die höchsten eta-Werte bis zu 0,43 erreicht; bei den Skalen zu Anforderungen und zur Unsicherheit des Arbeitsplatzes Werte von etwa 0,3 . Am schwächsten hängen die Aspekte aus dem Bereich soziale Beziehungen und Führung (eta in der Regel etwa 0,2) und die Belastungsfolgen $(0,14-0,19)$ mit der Berufsgruppe zusammen.
Diskussion. Während die JEM bei Gefahrstoffen oder auch bei einigen psychosozialen Themen wie Einfluss oder emotionale Anforderungen eine gute oder vertretbare Abschätzung der Belastungen auf Basis des Berufs oder der Tätigkeit liefert, ist dies insbesondere für relevante Parameter im Bereich soziale Beziehungen und Führung nicht der Fall. Hier kann eine tragfähige Belastungsabschätzung nur durch die Analyse der betrieblichen Organisationseinheiten gelingen.

\section{Schlüsselwörter}

Job-Expositions-Matrix · Psychosoziale Faktoren · COPSOQ · Gefährdungsbeurteilung · Arbeitsepidemiologie

\section{Are reference data from the COPSOQ database suitable for a JEM on psychosocial factors at work?}

\section{Abstract}

Background. The assessment of exposure through occupation or activities by using a job exposure matrix (JEM) is a proven process in many areas of work epidemiology.

Objective. The present study examined whether and to what extent such an assignment can also be successful in the field of psychosocial factors.

Material and methods. Based on 10,000 profession-representative cases from the COPSOQ database, the variance in 25 constructs on demands, influence and development possibilities, social relations and leadership, job insecurity and the outcome factors was analyzed in relation to the occupation classification (KIdB92).
Results. The strength of the relationship of psychosocial workplace factors to occupation is dependent on the subject area and the relationships are in the range of moderate to weak (eta coefficient $=0.11-0.43$ ). For the field of influence and development possibilities, the highest eta values up to 0.43 are reached, for the scales regarding demands and job insecurity values of approximately 0.3 result and the weakest relationship to the occupational group is found for constructs on social relations and leadership (eta values of approximately 0.2 ) and for outcome factors (0.14-0.19).

Discussion. While for hazardous substances or even some psychosocial topics, such as influence or emotional requirements, the JEM provides a good or justifiable estimate of the exposure based on occupation or activity, this is not the case in particular for relevant parameters in the area of social relations and leadership. Here a viable assessment of exposure and stress can only be achieved by analyzing the organizational units at the workplace.

\section{Keywords}

Job exposure matrix - Psychosocial factors · COPSOQ - Risk assessment - Work epidemiology
Diese Faktoren sind aber zudem sehr wichtig im Belastungs-BeanspruchungsGeschehen: Zum Bespiel weist die Skala Führungsqualität in Korrelations- und Regressionsmodellen mit dem COPSOQ den stärksten Zusammenhang zur Arbeitszufriedenheit auf $[6,7]$. Viele relevante Faktoren des psychosozialen Belastungsgeschehens lassen sich damit nicht oder nur sehr unzureichend in einer JEM abbilden.

Grund hierfür ist, dass viele psychosoziale Faktoren nicht primär vom Be- ruf („,job“) oder der Tätigkeit beeinflusst werden, sondern vom konkreten Arbeitsplatz („workplace“). Faktoren wie Gemeinschaftsgefühl oder Führungsqualität sind primär „workplace-related“, und können daher nicht durch eine Tätigkeitsmatrix abgebildet werden.

Dies deckt sich mit den Befunden von Schwartz et al. [8], Johnson et al. [2], und Bültmann et al. [1]. Durchgängig sind psychische Faktoren weniger berufsdeterminiert als physische und innerhalb der psychischen Faktoren zeigen sich die geringsten Zusammenhänge der Tätigkeit mit denjenigen Aspekten, die dem Bereich soziale Beziehungen und Führung zuzuordnen sind. Willert et al. [9] zeigten, dass für den Bereich soziale Beziehungen und Führung die Varianzaufklärung durch betriebliche Organisationseinheiten höher ist als die durch Tätigkeitsgruppen.

Der schwächere Zusammenhang der Konstrukte im Bereich soziale Beziehungen und Führung zur Berufsgruppe lässt sich - zumindest für unsere COPSOQ- 
Analysen - im Übrigen nicht durch niedrigere Gesamtvarianz in diesen Parametern erklären: Die Standardabweichungen für Einfluss mit 23 und für Führungsqualität mit 25 sind durchaus vergleichbar, aber die Varianz bei Führungsqualität liegt eben nicht auf der Berufsgruppe, sondern auf berufsunabhängigen Faktoren.

\section{Schlussfolgerungen}

Bei vielen technischen oder physischen Faktoren (Lasten, Lärm, Hitze etc.) und auch einigen psychosozialen Faktoren (Einfluss, Entwicklungsmöglichkeiten, Emotionale Anforderungen etc.) kann die Abschätzung der Belastung von Personen über ihre Tätigkeit mittels einer JEM funktionieren. Hierzu müssen die psychosozialen Faktoren aber detailliert und nicht summarisch erfasst werden.

Für die weniger mit der Tätigkeitsgruppe („job-related“), sondern eher mit dem konkreten Arbeitsplatz (,workplace-related“; direkte Vorgesetze, Kollegen) variierenden Faktoren kann eine JEM keine hinreichend verlässlichen Ergebnisse liefern.

JEM zu Gefahrstoffen oder physischen Expositionen können oftmals durch eine genauere Klassifizierung der Tätigkeiten (Homogenisierung) verbessert und verfeinert werden.

Im Fall der psychosozialen Faktoren im Bereich „workplace-related“ ist die geringe Varianzaufklärung nach unserer Einschätzung aber nicht darauf zurückzuführen, dass die Tätigkeit nicht genau genug klassifiziert wäre, sondern grundsätzlicher Natur: Wenn eine Belastung nicht mit der Tätigkeit zusammenhängt, hilft auch eine genauere Aufschlüsselung der Tätigkeit nicht weiter. Hier sollten aber weitere Analysen erfolgen, um zu prüfen, ob diese Vermutung zutrifft.

In Hinblick auf die arbeitsepidemiologische Forschung und auf die umfassende betriebliche Gefährdungsbeurteilung psychischer Faktoren bedeutet das, dass viele relevante Themen einer Abschätzung durch eine JEM nicht zugänglich sind.

Die Formulierung im Arbeitsschutzgesetz ( $\$ 5,2$, ArbSchG) „Der Arbeitgeber hat die Beurteilung ja nach Art der
Tätigkeiten vorzunehmen. Bei gleichartigen Arbeitsbedingungen ist die Beurteilung eines Arbeitsplatzes oder einer Tätigkeit ausreichend" muss auf der Basis dieser Befunde für die psychischen Faktoren aus unserer Sicht so aufgefasst werden, dass hier auch bei gleicher Berufsgruppe oder Tätigkeit keine gleichen Arbeitsbedingungen vorliegen (müssen) und insofern bei der psychischen Gefährdungsbeurteilung die Belastungen bei allen Beschäftigten ermittelt werden müssen.

\section{Korrespondenzadresse}

\section{Dr. M. Nübling}

Freiburger Forschungsstelle für Arbeitswissenschaften GmbH (FFAW)

Bertoldstr. 63, 79098 Freiburg, Deutschland nuebling@ffaw.de

\section{Einhaltung ethischer Richtlinien}

Interessenkonflikt. M. Nübling, M. Vomstein, A. Haug und $\mathrm{H}$.-J. Lincke geben an, dass kein Interessenkonflikt besteht.

Dieser Beitrag beinhaltet keine von den Autoren durchgeführten Studien an Menschen oder Tieren.

Open Access. Dieser Artikel wird unter der Creative Commons Namensnennung 4.0 International Lizenz (http://creativecommons.org/licenses/by/4.0/deed. de) veröffentlicht, welche die Nutzung, Vervielfältigung, Bearbeitung, Verbreitung und Wiedergabe in jeglichem Medium und Format erlaubt, sofern Sie den/die ursprünglichen Autor(en) und die Quelle ordnungsgemäßnennen, einen Linkzur Creative Commons Lizenz beifügen und angeben, ob Änderungen vorgenommen wurden.

\section{Literatur}

1. Bültmann U, Kant I, van Amelsvoort LG, van den Brandt PA, KasI SV (2001) Differences in fatigue and psychological distress across occupations: results from the Maastricht Cohort Study of Fatigue at Work. JOccup Environ Med 43(11):976-983

2. Johnson JV, StewartW, Fredlund P, Hall EM, Theorell $T$ (1990) Psychosocial job exposure matrix: an occupationally aggregated attribution system for work environment exposure characteristics. National Institute for Psychosocial Factors and Health, Stockholm

3. Kristensen TS, Hannerz H, Høgh A, Borg V (2005) The Copenhagen Psychosocial Questionnaire (COPSOQ). A tool for the assessment and improvement of the psychosocial work environment. Scand JWork Environ Health 31(6):438-449

4. Kroll LE (2011) Konstruktion und Validierung eines allgemeinen Index für die Arbeitsbelastung in beruflichen Tätigkeiten anhand von ISCO-88 und KIdB-92. Methoden Daten Anal 5(1):63-90
5. Nübling M, Stößel U, Hasselhorn HM, Michaelis M, Hofmann F (2005) Methoden zur Erfassung psychischer Belastungen - Erprobung eines Messinstrumentes (COPSOQ). Schriftenreihe der Bundesanstalt für Arbeitsschutz und Arbeitsmedizin, Fb 1058. Wirtschaftsverlag NW, Bremerhaven

6. Nübling M, Stößel U, Michaelis M (2010) Messung von Führungsqualität und Belastungen am Arbeitsplatz. Die deutsche Standardversion des COPSOQ (Copenhagen Psychosocial Questionnaire). In: Badura B, Schroder H, Klose J, Macco M (Hrsg) Fehlzeiten-Report 2009. Schwerpunktthema:Arbeit und Psyche: Belastungen reduzieren -Wohlbefinden fordern. Springer, Berlin, S79-87

7. Nübling $M$, Seidler $A$, Garthus-Niegel $S$, Latza $U$, Wagner M, Hegewald J, Liebers F, Jankowiak S, Zwiener I, Wild P, Letzel S (2013) The Gutenberg Health Study: measuring psychosocial factors at work and predicting health and work-related outcomes with theERI and the COPSOQ questionnaire. BMC Public Health 13:538

8. Schwartz JE, Pieper CF, Karasek RA (1988) A procedure for linking psychosocial job characteristics data to health surveys. Am J Public Health 78:904-909

9. Willert MV, Basinas I, Schliinssen V, Grynderup MB, Stokholm ZA, Bonde JP, Thomsen JF, Vammen MA, Hansen ÄM, Andersen JH, Rugulies R, Kaerlev L, Kolstad H (2016) Grouping strategies for psychosocial work exposures. Occupational health: think globally, act locally. Epidemiology in Occupational Health Conference (25th EPICOH), Barcelona, S 112 (Oral session 24, Exposure assessment methods, Book of Abstracts) 